\title{
Notes on the hybrid URANS/LES turbulence modeling for Internal Combustion Engines simulation
}

\author{
V. K. Krastev ${ }^{\mathrm{a}, *}$, G. Di Ilio ${ }^{\mathrm{b}}$, G. Falcucci ${ }^{\mathrm{c}}$, G. Bella ${ }^{\mathrm{c}}$ \\ ${ }^{a}$ Department of Economics, Engineering, Society and Business Organization, University of Tuscia, Largo dell'Università snc 01100 Viterbo, Italy \\ ${ }^{b}$ Department of Engineering, University of Rome 'Niccolò Cusano', Via don Carlo Gnocchi 3, 00166 Rome, Italy \\ 'Department of Enterprise Engineering 'Mario Lucertini', University of Rome 'Tor Vergata', Via del Politecnico 1, 00133 Rome, Italy
}

\begin{abstract}
In the past 20 years, Large Eddy Simulation methods have continuously increased their popularity among the Internal Combustion Engines modeling community, due to their intrinsic potential in the description of the unsteady and randomly generated in-cylinder flow structures. Such capability has gained further relevance in the simulation of modern turbocharged GDI engines, where the high-fidelity resolution of cycle-to-cycle variability phenomena is crucial for the evaluation of the engine performance and emission trends. Nonetheless, even after many years of development the application of standard LES methods to full-scale engine geometries is still not straightforward, due to: the need for specific, turbulence-generating boundary conditions at open ends; more severe grid resolution/quality and time step requirements compared to unsteady RANS; the need for high-order (at least second-order accurate) numerical schemes. Therefore, a viable alternative might be found in hybrid URANS/LES turbulence modeling, which has the potential to achieve adequate scale-resolving levels wherever actually needed, but mitigating at the same time some of the aforementioned concerns. In the present work we discuss the current status and perspectives of URANS/LES hybrids in the ICE field, based on the scientific literature state-of-the art and on a series of previous computational studies made by the authors. Outcomes from this study essentially confirm that this class of methods deserve further attention and will likely support URANS and standard LES in the near future as an effective computational tool for the ICE development and optimization.
\end{abstract}

(C) 2018 The Authors. Published by Elsevier Ltd.

This is an open access article under the CC BY-NC-ND license (https://creativecommons.org/licenses/by-nc-nd/4.0/) Selection and peer-review under responsibility of the scientific committee of the 73rd Conference of the Italian Thermal Machines Engineering Association (ATI 2018).

\footnotetext{
* Corresponding author. Tel.:+39 0761357676; .

E-mail address: v.krastev@unitus.it; v.krastev84@gmail.com
} 


\section{Review of hybrid URANS/LES methods for ICE flow modeling}

Hybrid URANS/LES turbulence models have been steadily developed for engineering applications since 1997 [1] and, at present, are considered a reliable and efficient option for fluid flow problems in the aerospace and turbomachinery fields $[2,3]$. On the other hand, the first noticeable application of such class of methods to Internal Combustion Engines is much more recent, as it dates back to 2009 [4]. Reasons for such a late development might be classified as geometry-dependent (e. g. complex moving boundaries and very small gaps between adjacent moving walls) or physics-dependent (continuously varying Reynolds number, simultaneous presence of wallimpinging and tangential flows, generation-disruption of large-scale flow structures [5]). In spite of this, in recent years the interest in URANS/LES hybrids for ICE modeling has marked a significant growth [6], essentially due to the need for scale-resolving-capable computational tools which might be regarded as alternative to standard LES approaches.

A comprehensive and detailed discussion about the currently available hybrid formulations has already been done in previous excellent reviews (see e. g. [7,8]) and is, therefore, out of the scope of the present work. Instead, to regroup the ICE-related hybrid modeling literature we propose two simple classification criteria (see Table 1): first, we divide Detached Eddy Simulation (DES) based methodologies from non-DES ones; second, each methodology is then classified as seamless or zonal. Following the definition found in [8], we call seamless (or universal) all models that are able to switch automatically from one method (URANS) to the other (LES), resulting in a change in terms of frequency and wave number resolutions. Conversely, zonal methods adopt pre-defined pure-URANS, pure-LES or seamless URANS/LES zones [8,9], in order to fully clarify the expected behavior of the numerical solution.

The first relevant seamless DES study on engine-like geometries is reported in the work of Hasse et al. [4], where a two-equation SST DES formulation was used to investigate the simplified tumble vortex compression described in [10]. In this work, the SST DES model was found to be competitive with LES for the capturing of the large-scale precessing tumble vortex and returned accurate mean-flow predictions of the intake flow motion. In a subsequent paper from the same authors [11], the same seamless DES model was adopted for the reproduction of the cold-flow cyclic variability measured in a real engine motored configuration. In this case, simulations were carried out on a relatively fine unstructured computational grid (up to $3 \cdot 10^{6}$ elements) and a relatively small number of engine cycles (up to 13) was considered for statistical computations. Results from this study showed a reasonable agreement with the experiments, but questioning at the same time the statistics validity due to the unavailability of a large number of statistical samples. Seamless DES was also applied in [12], using a time-scale based URANS formulation as the baseline model and stressing some of the issues related to the efficient URANS-to-LES switching in complex internal flow geometries. Further applications can be found in [13] and [14], where the seamless DES approach was additionally compared to LES and other hybrid methods, finding good overall performances in realistic engine geometries but confirming at the same time the uncertainties related to the standard DES switching mechanism [9].

Zonal-DES (ZDES) was initially introduced by S. Deck for aerospace engineering computations [15], with the aim of taking full advantage of each mode of operation (URANS, LES or various forms of seamless DES) across different parts of the computational domain. This kind of zonal splitting is potentially attractive for full-scale ICE simulation, since it allows to enable scale-resolving capabilities only where needed the most (in-cylinder volume), saving computational resources elsewhere (inlet/exhaust ducts, plenums, etc.). ZDES investigations of engine-like geometries are reported in [6], and [16-18], where a zonal reformulation of the same DES model proposed in [12] was applied together with the ZDES rearrangement of the RNG k- $\varepsilon$ URANS closure. Results from these studies show that a much better control of the solution behavior can be easily achieved with only minor modifications to any standard DES approach. However, questions like the near-wall behavior/treatment and the optimal URANS/LES interface treatment were not investigated in details. 
Besides DES, two additional seamless methodologies were extensively tested on ICE-relevant geometries in recent years. The first one is Menter's Scale-Adaptive Simulation (SAS) approach [19], which is directly derived from the SST k- $\omega$ URANS model. Differently from DES, in the SAS formulation there is no grid-dependent length scale switching and the only difference with the baseline URANS form is a source term in the $\omega$-equation that depends on the second spatial derivative of the velocity field. The source activates itself in the presence of smallscale instabilities in the flow, adjusting the eddy viscosity to a level that allows the formation of a turbulent spectrum. Otherwise, if the instabilities are too weak the model simply reverts back to the original URANS form. The computational studies found in [14] and [20-22] suggest that the SAS approach is fully capable of capturing large-scale unsteady flow structures, even in production-grade engine geometries. In addition, it seems to exhibit a more robust URANS-to-LES switching mechanism compared to seamless DES, which is desirable when there is limited a-priori knowledge about the optimal grid/numerical setup for the specific engine configuration. As a drawback, a more aggressive grid refinement strategy seems necessary in regions where a substantial scale-resolving capability is required. The last seamless approach analyzed here is called Dynamic Length-scale Resolution Method (DLRM) and is based on a local adaptive filtering operation that directly involves the Reynolds stress tensor produced by any URANS turbulence model [23]. The filter form is general and is designed to be consistent with the turbulence scaling laws in regions where the scale-resolving mode is enabled, while the URANS mode fallback is somewhat more conservative compared to the seamless DES. Results from the DLRM applications available in the literature $[23,24]$ show good scale-resolving capabilities and a robust mode of operation, even for coarse sub-milion computational grids.

Table 1. Relevant bibliography about hybrid URANS/LES applications to ICE flow modeling.

\begin{tabular}{lll}
\hline Model type & Reference & Year of publication \\
\hline DES (seamless) & {$[4],[11],[12]$,} & $2009,2010,2015$, \\
& {$[13],[14]$} & 2016,2017 \\
DES (zonal) & {$[6],[16],[17],[18]$} & $2018,2016,2017$, \\
& & 2017 \\
Others (seamless) & {$[14],[20],[21]$,} & $2017,2016,2016$ \\
& {$[22],[23],[24]$} & $2018,2015,2018$ \\
\hline
\end{tabular}

\section{Open issues and areas of improvement}

Based on the brief survey presented in the previous paragraph, we focus here on several key-points, which have not been fully addressed yet and/or need further development to extend the applicability of URANS/LES hybrids for comprehensive engine modeling and simulation.

\subsection{URANS-to-LES switching in seamless approaches}

Soon after its first appearance in 1997, it has been quickly detected that the original seamless DES switching formulation possesses an undesirable dependence on grid spacing, which might easily produce inconsistent flow features close to the walls or in the proximity of separation points [9]. Although subsequent modifications have found to significantly mitigate this problem through the introduction of semi-empirical delaying functions [25], the general validity of such type of correction for complex internal flows is debatable. Based on our previous experiences [12,16], the standard delaying function form proposed in [25] may produce substantially different results even for relatively simple geometrical configurations, depending on the local flow regime (see Fig. 1).

It is the authors' opinion that the other two seamless methods introduced in Section 1 possess a more consistent switching behavior compared to (standard) DES. More specifically, in agreement with the statements contained in [14] we consider the SAS SST model as a conceptually robust modeling choice for the scale-resolving simulation of realistic ICE configuration. However, it should be also underlined that all the computational studies found in the literature are characterized by very fine meshes $\left(8 \cdot 10^{6}\right.$ computational nodes and above), which poses the question of 
the actual scale-resolving performances of SAS in the case of relatively coarse, engineering-grade meshes. In addition, special attention must be given to the discretization of the velocity second derivatives, in order to properly capture the source term activation. Concerning DLRM, the proposed dynamic filtering procedure has shown very interesting results under coarse grid conditions, which would have produced highly unresolved results with any standard LES modeling option (see Fig. 2). However, the filter formulation partly relies on empirical arguments that have not been yet fully verified by the model developers under realistic engine operating conditions [27].

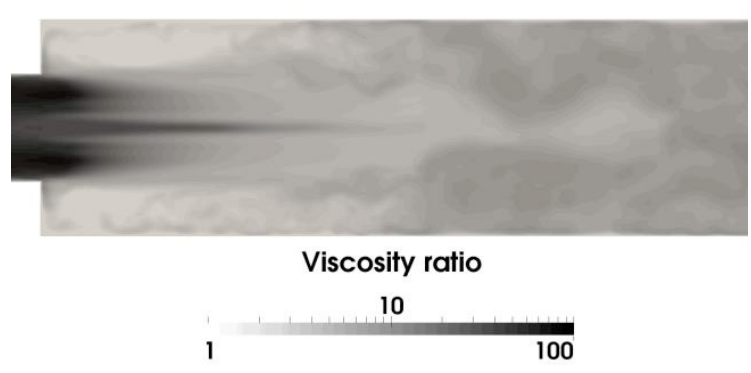

(a)

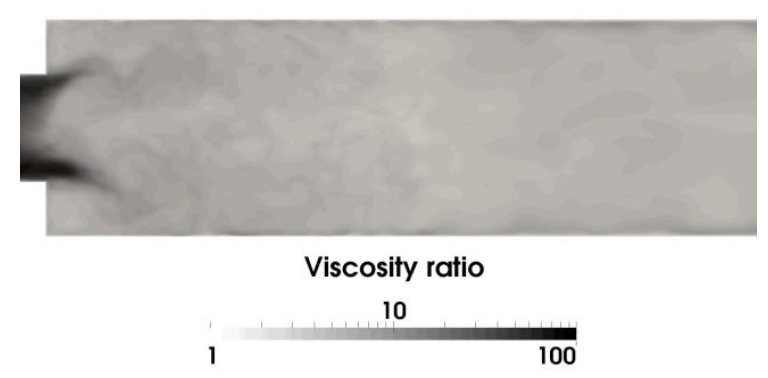

(b)

Fig. 1. Instantaneous viscosity ratio distribution during the DDES simulation of a reference abrupt expansion flow [26]: (a) zero-swirl case; (b) case with moderate swirl imposed at the inlet of the upstream pipe. Figure adapted from [12].

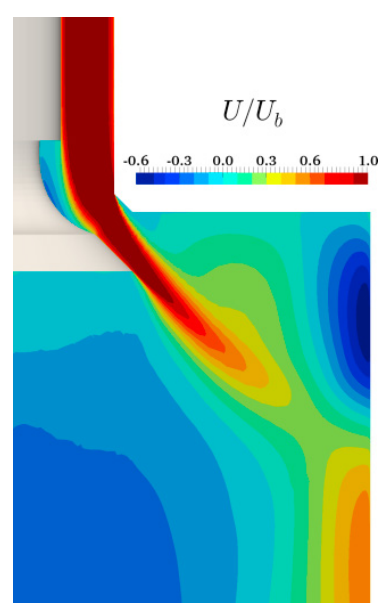

(a)

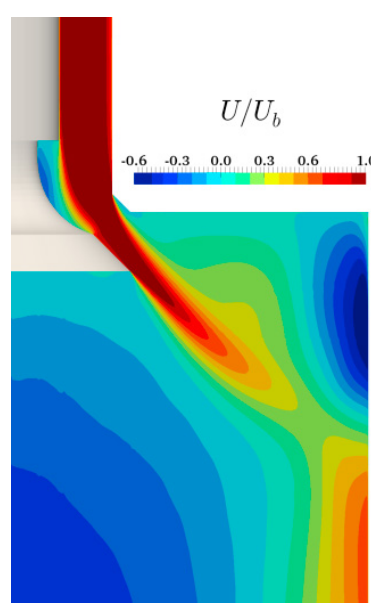

(b)

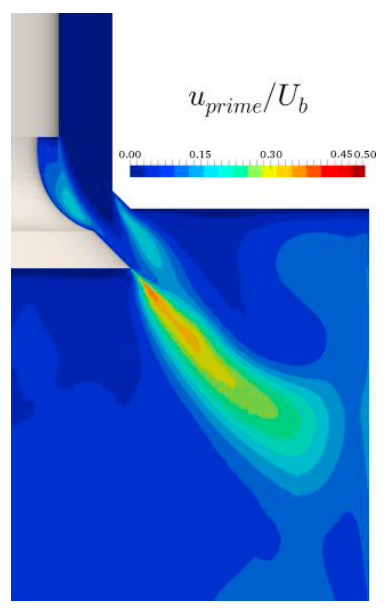

(c)

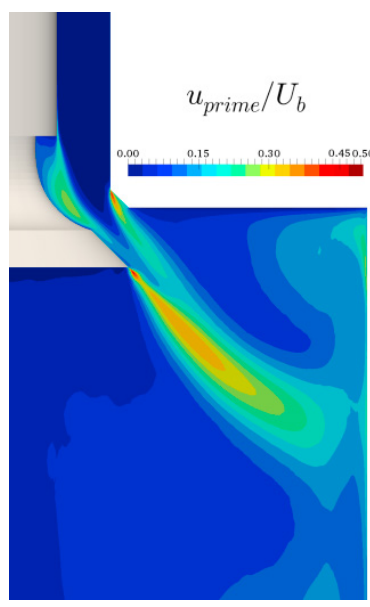

(d)

Fig. 2. Symmetry plane contours extracted from the DLRM simulation of a reference static intake valve geometry [28]: (a) mean axial velocity, coarse grid; (b) mean axial velocity, fine grid; (c) RMS axial velocity fluctuation, coarse grid; (d) RMS axial velocity fluctuation, fine grid. The coarse grid is made by $7 \cdot 10^{5}$ cells, the fine grid is made by $5.3 \cdot 10^{6}$ cells. Figure courtesy of Prof. F. Piscaglia [27].

\subsection{Interface treatment in zonal approaches}

According to S. Deck [15], in ZDES there is no need of a special treatment at the URANS/LES interface, provided that the upcoming flow in URANS mode has a sufficient amount of momentum to rapidly overwhelm the modeled turbulent content and trigger flow instabilities in the LES-treated region. However, this is no longer the case when the upcoming flow is characterized by a relatively low Reynolds number (see Fig. 3) and/or when complex two-way passages may occur across the interface. Different methods where developed in order to ensure resolved turbulence generation on the LES side and consistent interface coupling on the URANS side $[5,7,8,29,30]$. 
All such methods represent, however, a significant departure from the original ZDES elegance and simplicity and thus stimulating the debate on whether it should be better to focus on the refinement of seamless approaches rather than developing increasingly complex zonal methodologies. In that sense, the authors have no clear opinion, as our general belief is that both modeling families will maintain their own strengths and weaknesses, depending on the specific application and simulation targets.

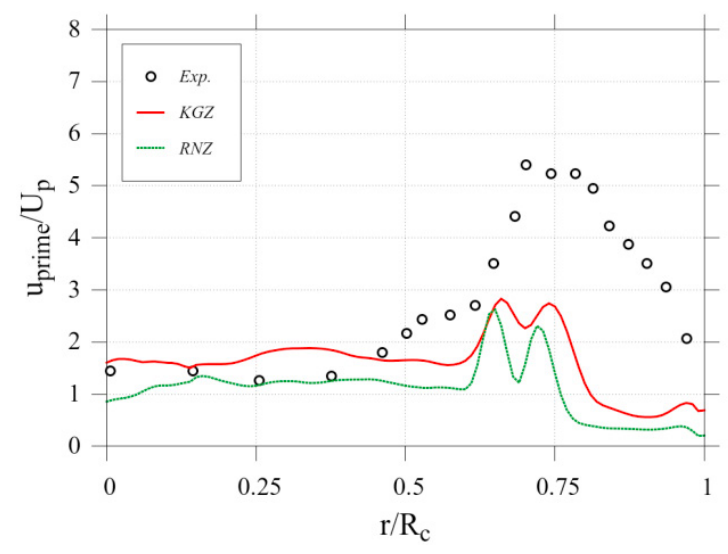

(a)

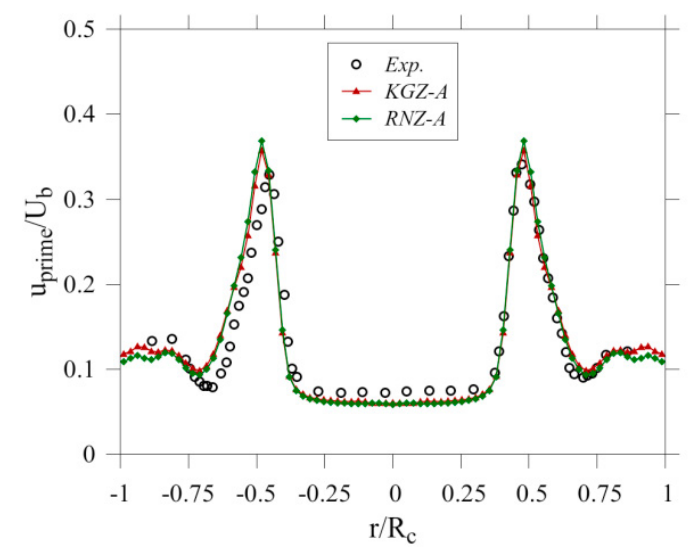

(b)

Fig. 3. RMS axial velocity fluctuation profiles obtained with two-equation ZDES models, for two different engine-like reference flow configurations: (a) low-speed piston/cylinder assembly [31], $\mathrm{z}=10 \mathrm{~mm}$ from the cylinder head during the intake stroke at the maximum piston speed conditions $\left(\mathrm{CA}=90^{\circ}\right)$, intake jet Reynolds number of $\sim 5 \cdot 10^{3}$; (b) fixed intake valve geometry [28], $\mathrm{z}=20 \mathrm{~mm}$ from the cylinder head, intake jet Reynolds number of $\sim 4 \cdot 10^{4}$. Figure adapted from [6].

\subsection{Wall treatment}

Near-wall resolution is and will most likely remain a major obstacle towards the usage of well-resolved LES in the ICE context. In fact, all the currently available ICE-relevant engineering-grade LES studies (see [32] for an effective definition of engineering LES) adopt wall functions or other types of wall modeling options in order to obtain a reasonable estimate of near-wall quantities keeping the computational requirements down to an acceptable level. It is important to note that, if the near-wall behavior of the flow solution is not fully clarified, the wall treatment issue is still there even in the hybrid URANS/LES framework. Indeed, almost all of the works reviewed in Section 1 (including the ones from the authors) do not present any rigorous analysis of the specific wall treatment adopted, focusing instead on the scale-resolving features in the bulk of the flow. More specifically, it seems to be a common practice to use the same wall-treatment options that would have been applied in conjunction with the underlying URANS model alone, which however is a consistent choice only if the boundary layers are completely managed by the URANS branch of the hybrid formulation. In a recent work about this topic [5], it is shown that coupling advanced RANS-based wall modeling with a zonal hybrid methodology, in which boundary layers are always covered by the URANS branch, might lead to very good near-wall flow predictions in the presence of engineering-grade mesh resolutions and without affecting the scale-resolved content in the core of the domain. Determining the best wall modeling candidate for seamless approaches is far less obvious and, in our opinion, should represent a major concern for the near-future development of seamless methodologies for ICE applications.

\subsection{Sub-models integration}

In spite of the large literature records on the integration between LES turbulence modeling and other physical/chemical sub-models [32], such as combustion and fuel spray models, the equivalent literature for URANS/LES hybrids is practically absent, especially among the ICE modeling community. In cases where the bulk of the flow is always (or mostly) treated in LES mode, such as with zonal or seamless DES, this means that 
integration tests should be made between the already available LES-adapted sub-models and the non-standard subgrid scale closure form that emanates from the underlying URANS framework (e. g. a two-equation SST or k- $\varepsilon$ SGS closure). In the SAS and DLRM cases, where the URANS/LES scales separation changes continuously across the spatial and temporal domains, sub-models integration might become a much more subtle task, due to the likelihood of simultaneous coexistence of URANS and LES fields at a one-cell (or one-time-step) distance one from another.

\section{Conclusions}

Far from being an exhaustive review of all the conceptual and practical aspects of efficient hybrid URANS/LES turbulence modeling implementation, the present work aims at returning a brief snapshot of the current status of such kind of modeling practice in the ICE multidimensional simulation community.

Our analysis has been divided into four specific modeling areas, namely: the switching mechanism in seamless approaches; the interface treatment in zonal approaches; the wall treatment; the sub-models integration. Based on the literature survey and on our previous experiences, the first three areas can be further classified as methodological aspects. Methods and approaches are already available, but none of them is undoubtedly the best one in every possible situation. It is, therefore, reasonable to assume that these areas are likely to steadily improve in the next years, thanks to an increasingly larger application database that will allow refining the existing options. Conversely, the integration between turbulence modeling and other physical sub-models (fuel sprays, combustion, etc.) is a more subtle task, which has to be considered as belonging to the conceptual aspects family. More specifically, we believe that the achievement of a consistent and simultaneous sub-models coupling with the URANS (i. e. statistically modeled) and LES (i. e. instantaneously and locally filtered) portions of the computational domain will require significant theoretical development efforts in the near to mid-term future.

Broadly speaking, it is authors' opinion that such class of methods has not fully undisclosed its full potential yet and we hope, therefore, that the points and issues covered here might represent a useful basis for other researchers interested in the discussion and development of this topic.

\section{Acknowledgements}

The authors would like to kindly acknowledge Prof. Federico Piscaglia for the useful comments and data exchange about the DLRM approach development and validation.

\section{References}

[1] Spalart, P. R., Wou, W. H., Strelets, M., and Allmaras, S. R. "Comments on the feasibility of LES for wings, and on a hybrid RANS/LES approach", in C. Liu and Z. Liu (eds) Advances in DNS/LES, (1997) Greyden Press, USA.

[2] Tucker, P. G. "Computation of unsteady turbomachinery flows: Part 2-LES and hybrids." Prog. Aerosp. Sci. 47 (2011): 546-569, doi:10.1016/j.paerosci.2011.07.002.

[3] Tucker, P. G., and DeBonis, J. R. "Aerodynamics, computers and the environment." Philos. Trans. R. Soc. A 372 (2014), doi:10.1098/rsta.2013.0331.

[4] Hasse, C., Sohm, V., and Durst, B. "Detached Eddy Simulation of Cyclic Large Scale Fluctuations in a Simplified Engine Setup." International Journal of Heat and Fluid Flow 30 (2009): 32-43, doi:10.1016/j.ijheatfluidflow.2008.10.001.

[5] Keskinen, K., Nuutinen, M., Kaario, O., Vuorinen, V., Koch, J., Wright, Y. M., Larmi, M., and Boulochos, K. "Hybrid LES/RANS with wall treatment in tangential and impinging flow configurations." International Journal of Heat and Fluid Flow 65 (2017): 141-158, doi: 10.1016/j.ijheatfluidflow.2017.04.001.

[6] Krastev, V. K., Silvestri, L., and Bella, G. "Effects of Turbulence Modeling and Grid Quality on the Zonal URANS/LES Simulation of Static and Reciprocating Engine-Like Geometries." SAE Technical Paper 2018-01-0173 (2018), doi:10.4271/2018-01-0173.

[7] Fröhlich, J., and von Terzi, D. "Hybrid LES/RANS methods for the simulation of turbulent flows." Prog. Aerosp. Sci. 44(5) (2008): 349-377, doi:10.1016/j.paerosci.2008.05.001.

[8] Sagaut, P., Deck, S., and Terracol, M. Multiscale and Multiresolution Approaches in Turbulence - LES, DES and Hybrid RANS/LES Methods: Applications and Guidelines ( $2^{\text {nd }}$ ed.), (2013) Imperial College Press, London, UK.

[9] Spalart, P. R. "Detached-Eddy Simulation." Annu. Rev. Fluid Mech. 41 (2009): 181-202, doi:10.1146/annurev.fluid.010908.165130.

[10] Boreé, J., Maurel, S., and Bazile, R. "Disruption of a compressed vortex." Phys. Fluids. 14(7) (2002): 2543-2556, doi:10.1063/1.1472505.

[11] Hasse, C., Sohm, V., and Durst, B. "Numerical Investigation of Cyclic Variations in Gasoline Engines Using a Hybrid URANS/LES Modeling Approach" Computers \& Fluids 39 (2010): 25-48, doi:10.1016/j.compfluid.2009.07.001. 
[12] Krastev, V. K., Bella, G., and Campitelli, G. "Some Developments in DES Modeling for Engine Flow Simulation." SAE Technical Paper 2015-24-2414 (2015), doi:10.4271/2015-24-2414.

[13] Hasse, C. "Scale-resolving simulations in engine combustion process design based on a systematic approach for model development" International J of Engine Research 17(1) (2016): 44-62, doi:10.1177/1468087415597842.

[14] Buhl, S., Dietzsch, F., Buhl, C., and Hasse, C. "Comparative study of turbulence models for scale-resolving simulations of internal combustion engine flows" Computers \& Fluids 156 (2017): 66-80, doi:10.1016/j.compfluid.2017.06.023.

[15] Deck, S. "Zonal-Detached-Eddy Simulation of the Flow Around a High-Lift Configuration" AIAA Journal 43(11) (2005): 2372-2384, doi:10.2514/1.16810.

[16] Krastev, V. K., and Bella, G. "A Zonal Turbulence Modeling Approach for ICE Flow Simulation." SAE International Journal of Engines 9(3) (2016), doi:10.4271/2016-01-0584.

[17] Krastev, V. K., Silvestri, L., Falcucci, G., and Bella, G. "A Zonal-LES Study of Steady and Reciprocating Engine-Like Flows Using a Modified Two-Equation DES Turbulence Model." SAE Technical Paper 2017-24-0030 (2017), doi:10.4271/2017-24-0030.

[18] Krastev, V. K., Silvestri, L., and Falcucci, G. "A Modified Version of the RNG k- $\varepsilon$ Turbulence Model for the Scale-Resolving Simulation of Internal Combustion Engines." Energies 10 (2017): 2116, doi:10.3390/en10122116.

[19] Menter, F., and Egorov, Y. "The scale-adaptive simulation method for unsteady turbulent flow predictions. part 1: theory and model description." Flow, Turbul Combust 85(1) (2010): 113-138, doi:10.1007/s10494- 010- 9264- 5.

[20] Buhl, S., Hartmann, F., and Hasse, C. "Identification of Large-Scale Structure Fluctuations in IC Engines Using POD-Based Conditional Averaging." Oil \& Gas Science and Technology - Rev. IFP 71(1) (2016): 1, doi:10.2516/ogst/2015021.

[21] Buhl, S., Gleiss, F., Köhler, M., et al. "A combined numerical and experimental study of the 3d tumble structure and piston boundary layer development during the intake stroke of a gasoline engine." Flow, Turbul Combust 98(2) (2016): 579-600, doi:10.1007/s10494-016-9754-1.

[22] Buhl, S., Hain, D., Hartmann, F., and Hasse, C. "A Comparative Study of Intake and Exhaust Port Modeling Strategies for Scale-Resolving Engine Simulations" International J of Engine Research 19(3) (2018): 282-292, doi:10.1177/1468087417707452.

[23] Piscaglia, F., Montorfano, A., and Onorati, A. "A Scale Adaptive Filtering Technique for Turbulence Modeling of Unsteady Flows in IC Engines." SAE International Journal of Engines 8(2) (2015): 426-436, doi:10.4271/2015-01-0395.

[24] Wu, Y., Montorfano, A., Piscaglia, F., and Onorati, A. "A Study of the Organized in-Cylinder Motion by a Dynamic Adaptive ScaleResolving Turbulence Model" Flow, Turbul Combust 100(3) (2018): 797-827, doi:10.1007/s10494-017-9881-3.

[25] Spalart, P. R., Deck, S., Shur, M. L., Squires, K. D., et al. "A new version of detached-eddy simulation, resistant to ambiguous grid densities." Theor. Comput. Fluid Dyn. 20 (2006): 181-195, doi:10.1007/s00162-006-0015-0.

[26] Dellenback, P. A., Metzger, D. E., and Neitzel, G. P. "Measurements in Turbulent Swirling Flow Through an Abrupt Axisymmetric Expansion." AIAA Journal 26(6) (1988): 669-681, doi:10.2514/3.9952.

[27] Piscaglia, F. Personal communications with the authors (2016).

[28] Thobois, L., Rymer, G., Soulères, T., and Poinsot, T. "Large-Eddy Simulation in IC Engine Geometries." SAE Technical Paper 2004-011854, (2004), doi:10.4271/2004-01-1854.

[29] Shur, M., Spalart, P., Strelets, M., and Travin, A. "A hybrid RANS-LES approach with delayed-DES and wall modelled LES capabilities." International Journal of Heat and Fluid Flow 29(6) (2008): 1638-1649, doi:10.1016/j.ijheatfluidflow.2008.07.001.

[30] Jakirlić, S., Kniesner, B., and Kadavelil, G. "On interface issues in LES/RANS coupling strategies: a method for turbulence forcing." $J$. Fluid Sci. Technol. 6(1) (2011): 56-72, doi:10.1299/jfst.6.56.

[31] Morse, A. P., Whitelaw, J. H., and Yanneskis, M. "Turbulent Flow Measurements by Laser-Doppler Anemometry in Motored PistonCylinder Assemblies." Journal of Fluids Engineering 101(2) (1979): 208-216, doi:10.1115/1.3448937.

[32] Rutland, C. J. "Large-Eddy Simulations for Internal Combustion Engines - A Review" International J of Engine Research 12(5) (2011): 421-451, doi:10.1177/1468087411407248. 